\section{Apoptosis pattern and alterations of expression of apoptosis-related factors of supporting cells in Kölliker's organ in vivo in early stage after birth in rats}

\author{
Jun Liu, ${ }^{1,2,3,4}$ Linbin Cai, ${ }^{1,2,3}$ \\ Yuanyuan He, 1,2,3 Jun Yang 1,2,3 \\ ${ }^{1}$ Department of Otorhinolaryngology \\ Head and Neck Surgery, Xinhua \\ Hospital, Shanghai Jiaotong University \\ School of Medicine, Shanghai \\ ${ }^{2}$ Ear Institute, Shanghai Jiaotong \\ University School of Medicine, Shanghai \\ ${ }^{3}$ Shanghai Key Laboratory of \\ Translational Medicine on Ear and Nose \\ diseases, Shanghai \\ ${ }^{4}$ Department of Otorhinolaryngology, \\ Renji Hospital, Shanghai Jiaotong \\ University School of Medicine, \\ Shanghai, China
}

\begin{abstract}
Kölliker's organ is a temporary but indispensable structure in the development of the cochlea. Supporting cells (SCs) within it release adenosine 5'-triphosphate (ATP), which may play a crucial role in cochlear development before the onset of hearing. To reveal the apoptosis of Kölliker's organ in new-born rats, we studied the morphological changes and expression of apoptosis-related factors during early postnatal development. We found SCs in Kölliker's organ decreased in number and changed in appearance along the cochlea apex-to-base gradient, and the expression of caspase-3, caspase-8, caspase- 9 and bcl-2 in Kölliker's organ of the cochlea fluctuated along the course of postnatal development, with an expression peak at postnatal day 3 . This study demonstrates a time-dependent degeneration of Kölliker's organ during postnatal cochlea development, which might be triggered by endogenous factors.
\end{abstract}

\section{Introduction}

Kölliker's organ, a transient epithelial structure present in the developing cochlea, was first recognized in 1863 by Albert von Kölliker. Although Kölliker's organ was described more than one century ago, its function is still unknown, especially in the period after sensory cell differentiation. ${ }^{1}$ Rodents do not have hearing till the postnatal 12-14 days, suggesting the functional and structural immaturity of the auditory organs after birth. ${ }^{2}$ Before the onset of hearing, Kölliker's organ, also known as the greater epithelial ridge, locates medial to the inner hair cells (IHCs) and contains columnar supporting cells (SCs). Tritsch et $a l .{ }^{3}$ observed that SCs within Kölliker's organ release adenosine 5'-triphosphate (ATP) spontaneously and rhythmically in the immature ear, which depolarizes the IHCs and initiates bursts of firing, representing an experience-independent neural activity. Also, it has been recently demonstrated that immature IHCs fire spontaneously and evoke action potentials until the onset of hearing. ${ }^{4}$ Like the spiral bundles, ${ }^{5}$ they play a role in auditory system development; however, the period during which this activity occurs has not been defined and the mechanisms controlling the onset and cessation of this activity are largely unknown. It is proposed that the release of ATP from Kölliker's organ presumably exerts a modulatory effect through increasing or decreasing firing in a dose-dependent manner. ${ }^{6}$ Therefore, in view of ATP release, Kölliker's organ plays an indispensable role in the cochlear maturation and development. Interestingly, following the opening of the external auditory canal and the onset of sound-evoked hearing activities, Kölliker's organ degenerates in the cochlea. Previous studies suggested that the SCs may progressively disappear through apoptosis during postnatal development.7,8 However, the pattern of disappearance has not been described in detail yet.

Apoptosis plays a very active role in development to remove cells to control the size and sculpt the shape of an organ, and can be initiated by signals from outside or inside a cell. ${ }^{9}$ Caspases, normally present in the inactive forms, play essential roles in apoptosis in many organs. ${ }^{10,11}$ However, alterations of expression of apoptosis-related factors of SCs in Kölliker's organ have not been reported. Despite the well-known morphology of the mature cochlea, the process and mechanism of the disappearance of Kölliker's organ are not yet fully understood. Therefore, our study focused on the apoptosis pattern and alterations of expression of apoptosis-related factors such as caspase-3, caspase-8, caspase-9, bcl-2 of SCs in Kölliker's organ in the early stage after birth in the rat cochlea in vivo. Our results provided evidence that the endogenous factors are likely to contribute to apoptosis of Kölliker's organ.
Correspondence: Jun Yang, Department of Otorhinolaryngology Head and Neck Surgery, Xinhua Hospital, Shanghai Jiaotong University School of Medicine, 1665 Kongjiang Rd, Shanghai, China.

Tel. +86.21.25078999.

E-mail: otology-xinhua@hotmail.com

Key words: Kölliker's organ; supporting cells; apoptosis; development.

Contributions: JL and LC contributed equally to the paper.

Conflict of interest: the authors declare no conflict of interest.

Acknowledgments: this work was supported by the National Natural Science Foundation of China (No.81170919, No. 81470689, No. 81600797), the project of Shanghai Municipal Science and Technology Commission (No.14DJ1400201, No. 14DZ2260300, No.2014CB541705, No.16XD1402200).

Special thanks to Prof. Huawei Li and his laboratory members of Eye and ENT Hospital of Fudan University, for their technical support and scientific advice.

Received for publication: 29 July 2016. Accepted for publication: 21 June 2017.

This work is licensed under a Creative Commons Attribution-NonCommercial 4.0 International License (CC BY-NC 4.0).

(C) Copyright J Liu et al., 2017

Licensee PAGEPress, Italy

European Journal of Histochemistry 2017; 61:2706 doi:10.4081/ejh.2017.2706

\section{Materials and Methods}

\section{Animals and materials}

Male and female Sprague-Dawley rats at postnatal 1 to 14 days (P1, P3, P5, P7, $\mathrm{P} 10, \mathrm{P} 12$ and P14) were provided by the Shanghai Laboratory Animal Center of the Chinese Academy of Sciences (6 rats in each age group). All animal experiments were approved by Shanghai Jiaotong University School of Medicine, and the experimental methods were carried out in accordance with the approved guidelines by Institutional Animal Care and Use Committee of Shanghai Jiaotong University School of Medicine (License No. (Shanghai): 2008-0052). All chemicals were purchased from Sigma-Aldrich (St. Louis, MO, USA), unless otherwise stated. 


\section{Isolation of cochlear tissue}

Rats were anesthetized by an intraperitoneal injection of $10 \%$ chloral hydrate $(0.2$ $\mathrm{mL} / 100 \mathrm{~g}$ ), approved by the Animal Care Committee. The cochlea was dissected from the temporal bone, and then the otic capsule was carefully removed in a dish containing $0.01 \mathrm{M}$ sodium phosphate buffered saline (PBS). Auditory epithelium and underlying basilar membrane were isolated by removing the stria vascularis, spiral ligament and spiral ganglions, and used for further analysis.

\section{Basilar membrane immunofluorescence}

The cochleae of Sprague-Dawley rats at P1 were dissected from the temporal bone as described above, and then were fixed with 4\% paraformaldehyde solution for 30 min at room temperature. Then the basilar membranes were isolated and thoroughly rinsed with $0.01 \mathrm{M}$ PBS. We modified the protocol of immunofluorescence as described in our previous studies. ${ }^{12,13}$ They were in a blocking/permeabilization solution [10\% normal donkey serum (Jackson Labs, Bar Harbor, ME, USA) and 0.3\% Triton X-100 in $0.01 \mathrm{M}$ PBS] for $30 \mathrm{~min}$ at room temperature. They were subsequently incubated with goat anti-myosin VIIa (1:300, Proteus BD BioSciences, San José, CA, USA) and rabbit anti-Sox2 (1:200, Santa Cruz Biotechnology, Santa Cruz, CA, USA) antibodies in 5\% normal donkey serum and $0.1 \%$ Triton $\mathrm{X}-100$ in $0.01 \mathrm{M}$ PBS overnight at $4{ }^{\circ} \mathrm{C}$, mediated by DyLight 594 and DyLight 488 conjugated secondary antibodies (goat anti-rabbit IgG or rabbit anti-goat $\operatorname{IgG}$ ) at a concentration of 1:500 for $1 \mathrm{~h}$ at room temperature. Observation results were recorded under a fluorescence microscope after sealing slices. Images were photographed on a laser confocal scanning microscope (LSM 710, Zeiss, Jena, Germany) with a $20 \times$ lens by using Zeiss ZEN 2010 software. Images were processed using Adobe Photoshop software.

\section{Histological staining}

Postnatal rats at different developmental stages (P1, P5, P12) were fixed initially by intracardiac perfusion with ice-cold $4 \%$ paraformaldehyde in $0.1 \mathrm{M}$ phosphate buffer $(\mathrm{pH} 7.4)$ and the cochlea was then quickly removed. The round and oval windows of the cochlea were opened, and a small hole made in the bony apex of the cochlea. After perilymphatic perfusion with the above fixative, cochleae were post-fixed in the same fixative for $35 \mathrm{~min}$ at room temperature. P5 and older animals were decalcified in $10 \%$ EDTA at $\mathrm{pH}$ 7.4. The cochleae were thoroughly rinsed with $0.01 \mathrm{M}$ PBS and subsequently placed in a gradient of sucrose $15 \%$ for $3 \mathrm{~h}$ and $30 \%$ overnight. The cochleae were then embedded in optimum cutting temperature compound (OCT) at $4^{\circ} \mathrm{C}$ for $3 \mathrm{~h}$. Serial $8-\mu \mathrm{m}$-thick sections were cut with a cryostat microtome. Stain nuclei with $75 \%$ alum hematoxylin for $4 \mathrm{~min}$. After being rinsed in running tap water, the sections were differentiated with $0.3 \%$ acid alcohol. Then, the sections were rinsed in running tap water for $10 \mathrm{~min}$ and stained with $0.15 \%$ eosin for $2 \mathrm{~min}$; dehydrated in $95 \%$ and absolute alcohols, two changes of 2 min each; cleared in xylene, two changes of 2 min each, and mounted in Permount. Samples including the next two parts were observed under a light microscope (BX43; Olympus, Tokyo, Japan) with a $40 \times$ lens by using cell Sens software. Images were processed using Adobe Photoshop software.

\section{Immunohistochemical staining}

Slides of rat cochlear were made of in the same way as histological staining. Antigen retrieval was performed in $0.01 \mathrm{M}$ citrate buffer using a microwave for $2 \mathrm{~min}$. Then they were treated with $3 \% \mathrm{H}_{2} \mathrm{O}_{2}$ for 10 min and washed with $0.01 \mathrm{M}$ PBS. They were in a blocking/permeabilization solution $[10 \%$ normal donkey serum (Jackson Labs) and $0.3 \%$ Triton $\mathrm{X}-100$ in $0.01 \mathrm{M}$ $\mathrm{PBS}$ ] for $30 \mathrm{~min}$ at room temperature, followed by incubation with rabbit anti-caspase 3 antibody (1:200; Abcam, Cambridge, UK) or with rabbit anti-bcl2 polyclonal antibody $\quad(1: 200 ; \quad$ Santa Cruz Biotechnology) at $4{ }^{\circ} \mathrm{C}$ overnight, biotinylated rabbit anti-goat $\operatorname{IgG} \quad(1: 300$; BosterBio, Pleasanton, CA, USA) secondary antibody at $37^{\circ} \mathrm{C}$ for $30 \mathrm{~min}$, and streptavidin-biotin complex at $37^{\circ} \mathrm{C}$ for $30 \mathrm{~min}$. The following steps were the same as staining nuclei, dehydrating and mounting in Histological staining.

\section{TUNEL assay}

The sections were carefully prepared, and the apoptotic cells were observed using a TdT-mediated dUTP-biotin nick-end labelling (TUNEL) test system (Kit 11684795910; Roche, Indianapolis, IN, USA) according to the manufacturer's instructions. The sections were firstly incu- bated in permeabilisation solution $(0.1 \%$ Triton $\mathrm{X}-100,0.1 \%$ sodium citrate) for 2 min on ice $\left(2^{\circ} \mathrm{C}\right.$ to $\left.8^{\circ} \mathrm{C}\right)$. After rinsing slides with PBS twice, we added $50 \mu \mathrm{L}$ TUNEL reaction mixture $(5 \mu \mathrm{L}$ Enzyme solution and $45 \mu \mathrm{L}$ Label solution) and incubated for 60 min at $37^{\circ} \mathrm{C}$ in a humidified atmosphere in the slip during incubation. Following basilar membrane immunofluorescence, the apoptotic features of cell death were examined via a laser confocal scanning microscopes (LSM 710, Zeiss, Jena, Germany) with a $20 \times$ lens by using Zeiss ZEN 2010 software.

\section{Quantitative PCR}

RNA samples were extracted from the cochlea tissue containing Kölliker's organ using TRIzol (Invitrogen, Carlsbad, CA, USA). Every experiment was repeated six times; $1 \mu \mathrm{g}$ of total RNA was used in the first strand cDNA synthesis with TaqManH Reverse Transcription Reagents (Takara Bio Inc., Otsu, Shiga, Japan). Annealing temperature was optimized for each primer, which was listed on Table 1. The amplification was carried out in triplicate with the ABI PRISM 7500 Sequence Detection System (Applied Biosystem, Foster City, CA, USA), using SYBR green quantitative PCR mix (Takara Bio Inc.), and $\beta$-actin as the endogenous reference. Relative quantification of gene expression was calculated as the fold change using the $2^{\Delta \Delta \mathrm{Ct}}$ method based on Ct values for both target and reference genes.

\section{Western blotting analysis}

Proteins from the dissected cochlear tissue containing Kölliker's organ and organ of Corti were separated on SDS-PAGE, and transferred to immobilon polyvinyldifluoride membranes (Millipore, Billerica, MA, USA). The blots were blocked with 5\% nonfat dry milk in PBS-0.01\% Tween 20 (PBS-T) for $1 \mathrm{~h}$ at room temperature and then probed with antibodies against $\beta$-actin (Protein tech, 1:2000), bcl-2, caspase-3, caspase-8, caspase- 9 and cleaved caspase- 3 (Cell Signaling, Danvers, MA, USA; $1: 1000)$ for $1 \mathrm{~h}$ at room temperature and then overnight at $4^{\circ} \mathrm{C}$. After three times

Table 1. Primers for Realtime PCR.

\begin{tabular}{|c|c|c|}
\hline Gene & Forward & Reverse \\
\hline$\beta$-actin & 5'-CCGTAAAGACCTCTATGCCAACA-3' & 5'- GCTAGGAGCCAGGGCAGTAATC -3' \\
\hline caspase- 3 & 5'- GGAGCAGTTTTGTGTGTGTGAT -3' & 5'- GAAGAGTTTCGGCTTTCCAGT -3’ \\
\hline caspase-8 & 5'- ACGATATTGCTGAACGTCTGG -3’' & 5'- CCGACTGATATGGAAAAGCAG -3' \\
\hline caspase- 9 & 5' - AGCCAGATGCTGTCCCATAC -3' & 5'- CAGGAGACAAAACCTGGGAA -3' \\
\hline$b c l-2$ & 5'- ACCTGAATGACCACCTAGAG -3' & 5'- ACTTCCGACTGAAGAGTGAG -3' \\
\hline
\end{tabular}


washing with PBS-T buffer, the membranes were incubated with a secondary antibody [goat anti-rabbit $\operatorname{IgG}(1: 1000)$ or rabbit antimouse $\operatorname{IgG}(1: 2000)]$ for $1 \mathrm{~h}$ at room temperature. Following washing of the membrane, the immunoreactive bands were visualized by enhanced chemiluminescence (ECL) according to the instructions of the manufacturer using Bio-Rad ChemiDoc XRS+ (Bio-Rad Compony, USA).

\section{Statistical analysis}

Data analysis was performed with SPSS 20.0 software (IBM SPSS Inc., NewYork, NY, USA).

\section{Results}

\section{Immunofluorescence staining of} basilar membrane of $P 1$ rats

The contour of epithelial cell layer on basilar membrane of the cochlea in neonatal rats is shown in Figure 1. Hair cells were stained with Myosin-VIIa and all supporting cells including SCs within Kölliker's organ in the basilar membrane were stained with an antibody to Sox2, indicating an apex-to-base gradient of the SCs in Kölliker's organ. The number of SCs obviously decreased in the basal turn, compared with the apical.

\section{Morphological changes and the expression of caspases and bcl-2 in Kölliker's organ of the neonatal rats}

We assessed Kölliker's organ at postnatal day 1 , day 5 and day 12 (P1, P5, P12) in rats to confirm the morphological changes in the apical, middle, and basal turns of the cochlea (Figure 2). Kölliker's organ was evident throughout the turns, showing 3-5 rows in the base and 8-14 rows of SCs in the apex at P1. In P5 rats, Kölliker's organ in the basal turn became significantly smaller, while it reduced in the middle and the apical ones along with decreased number of SCs. P12 cochlea showed disappearance of the
SCs in Kölliker's organ with an apex-tobase gradient. The basal and the middle Kölliker's organ disappear, which was indicative of the maturity of the structure in P12 rats. However, the apical Kölliker's organ still existed in this stage, although the number of SCs in Kölliker's organ decreased and their morphology changed from high columnar to short columnar.

Immunohistochemistry and TUNEL (TdT-mediated dUTP nick-end labeling) stained sections from the apical, middle, and basal turns of the cochlea at P5 were displayed in Figure 3. TUNEL results showed that there were some apoptotic cells in Kölliker's organ because positive cells(green) were observed in the apical, middle, and basal turns of the cochlea (Figure 3 A-C). The bcl-2 protein was expressed in the cytoplasm of SCs in Kölliker's organ from the basal turn to the apex turn (Figure 3 D-F) at P5, which correlate with the apoptotic cells in the Kölliker organ. Likewise, the caspase-3 protein was expressed in the cytoplasm of
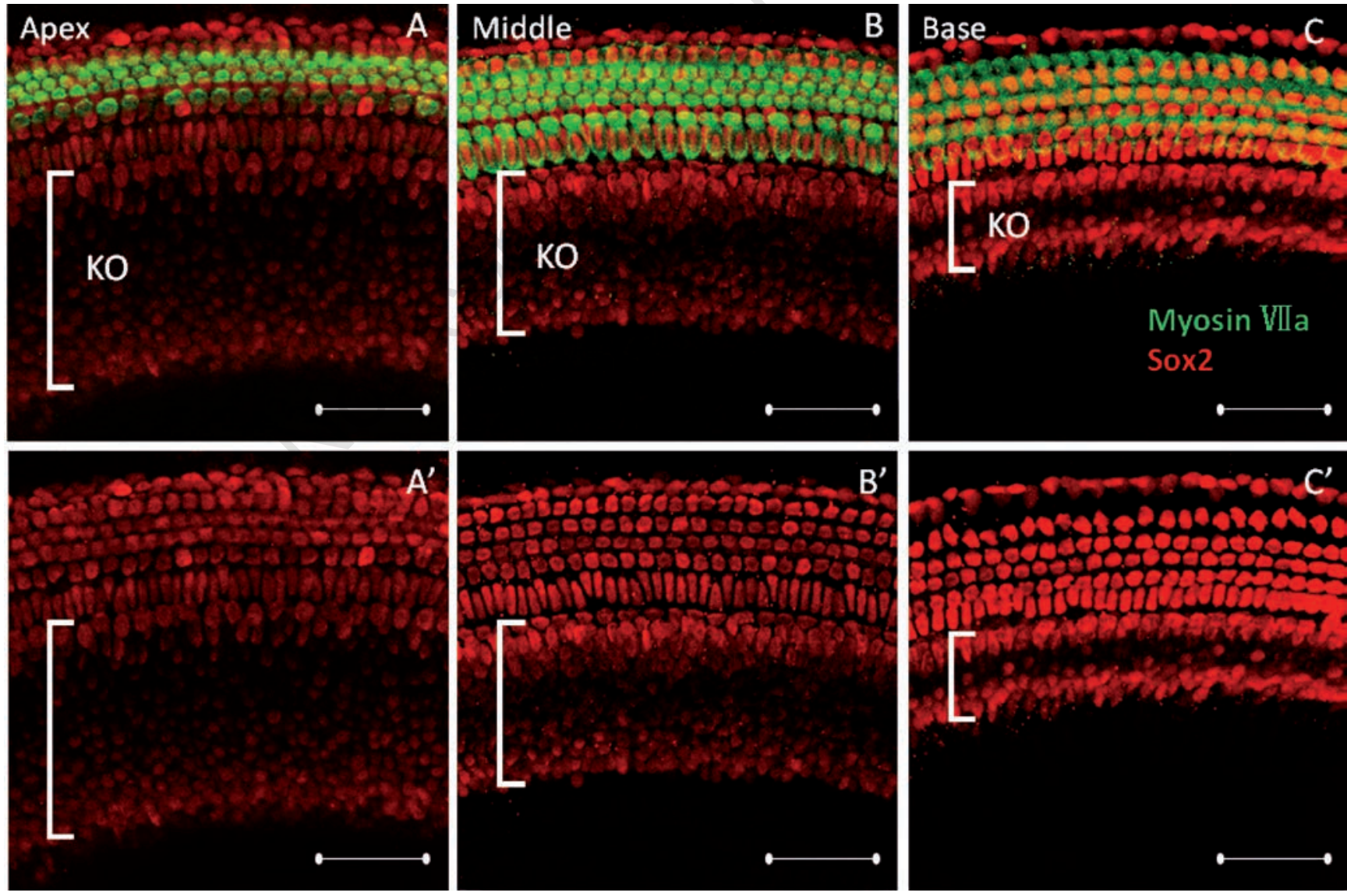

Figure 1. Immunofluorescence staining of basilar membrane of P1 rats. The apical (A), middle (B), and basal (C) turns were displayed. Myosin-VIIa labeled hair cells (green); Sox 2 marks all supporting cells, including Kölliker's supporting cells (red). The number of SCs in Kölliker's organ decreased with an apex-to-base gradient. Scale bars: $50 \mu \mathrm{m}$. 
SCs in Kölliker's organ from the basal to the apex turn at P5 (Figure $3 \mathrm{G}-\mathrm{I}$ ).

\section{Realtime PCR analysis of bcl-2,} caspase- 3 , caspase-8 and caspase-9 in Kölliker's organ in vivo

Quantitative PCR assay was used to analyze mRNA expression of caspase-3, caspase- 8 , caspase- 9 and bcl- 2 in the auditory sensory epithelium from $\mathrm{P} 1$ to $\mathrm{P} 14$ rats. The highest expression of bcl-2 was observed at P3 and P5 (2-2.5-fold of P1, $\mathrm{n}=6,{ }^{*} \mathrm{P}<0.05$ compared with $\mathrm{P} 1$, independent samples $t$-test) (Figure 4A). The expression of caspase- 3 and caspase- 8 peaked at P3, showing 1.8-fold and 2.5-fold compared to that at $\mathrm{P} 1\left(\mathrm{n}=6,{ }^{*} \mathrm{P}<0.05\right.$, independent samples $t$-test), then dropped to the $\mathrm{P} 1$ level, but caspase- 8 showed a further decrease at P14 ( 0.5 fold of $\mathrm{P} 1, \mathrm{n}=6$, $\# \mathrm{P}<0.05$ compared with $\mathrm{P} 1$, independent samples $t$-test) (Figure 4 B,C). The expression profile of caspase- 9 mRNA expression was different compared to caspase- 3 and caspase-8, showing low expression at P5, $\mathrm{P} 10$ and P12 (0.6-0.7-fold of the P1, n=6, $* \mathrm{P}<0.05$ compared with $\mathrm{P} 1, * * \mathrm{P}<0.05$ compared with $\mathrm{P} 1$, independent samples $t$-test) (Figure 4D).

\section{Western blot analysis of bcl-2,} caspase-3, cleaved caspase-3, caspase- 8 and caspase- 9 in Kölliker's organ in vivo

Afterwards, we analyzed the protein expression in the cochlear basilar mem- brane. Since caspase-3, caspase-8, caspase-9, and bcl-2 were located in the cytoplasm, we proceeded to fractionate the total protein into cytoplasmic and nuclear fractions. Cytoplasmic proteins were probed with each antibody, respectively (Figure 5). Consistent to their mRNA expression, bcl2 , caspase- 3 and caspase- 8 proteins were highest at P3, and caspase-9 expression showed a decrease at $\mathrm{P} 12$ and $\mathrm{P} 14$ without a peak expression. We also analyzed the protein expression of cleaved caspase-3, which consistently showed a higher expression in the cochlea of the $\mathrm{P} 3$ rats than that of P1 rats (Figure 6). The dynamic expression of apoptotic genes in the cochlea coincided with the morphological changes in Kölliker's organ.
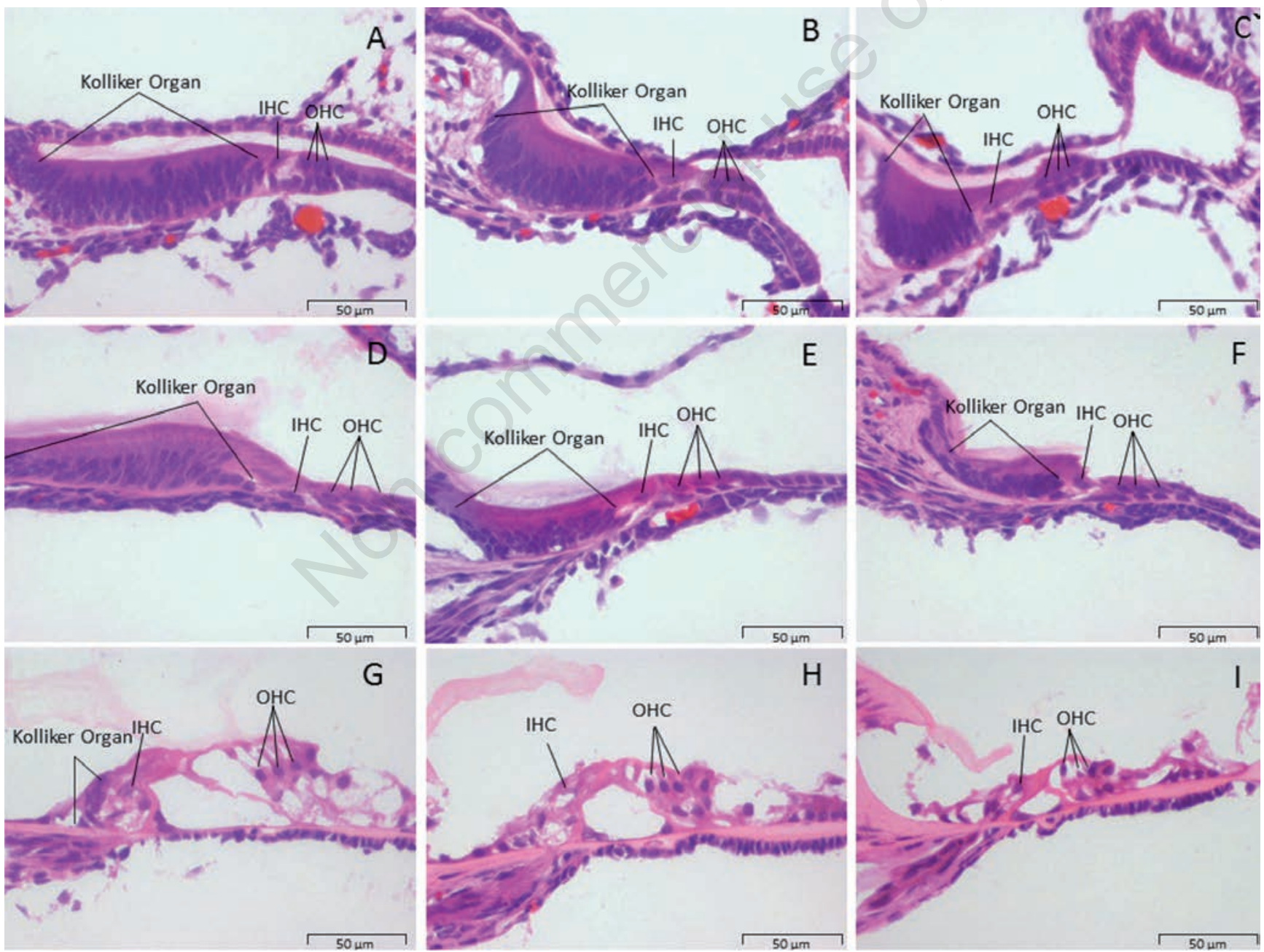

Figure 2. Histological staining of Kölliker's organ in neonatal rats. H\&E stained sections from the apical (A,D,G), middle (B,E,H), and basal (C,F,I) turns of the cochlea from P1, P5, and P12 were displayed. A-C) Kölliker's organ in P1 rat; Kölliker's organs were observed from apical to basal turn. D-F) Kölliker's organ in P5 rats, in which Kölliker's organ in the basal turn became significantly smaller, while it reduced along with decreased number of SCs. G-I) Kölliker's organ in P12 rat. Kölliker's organ in all turns except for the apical one disappeared, though the number of SCs decreased and their morphology changed from high columnar to short columnar. 


\section{Discussion}

Kölliker's organ develops first in the embryonic cochlear duct, but disappears at the onset of hearing in rodents, undergoing extensive remodeling. The differentiated Kölliker's organ is composed of numerous tightly packed, tall columnar epithelial cells filling the inner sulcus. ${ }^{14}$ As the cochlea matures, SCs within Kölliker's organ experiences a transformation, namely, columnar cells are replaced by cuboidal cells, forming the mature inner sulcus. ${ }^{14}$ This refinement likely involves apoptosis of columnar cells, ${ }^{8,14}$ though the exact mechanism behind it is largely unknown. It suggests a functional shift from genetically encoded process to an experience-dependent guidance. ${ }^{8,15}$ TUNEL results at P5 in our study confirmed that there was apoptosis of SCs in Kölliker's organ. However, few studies have focused on the apoptosis pattern of Kölliker's organ. To clarify this pattern, we observed the morphological changes in the developing cochlea, showing that the number of SCs in the Kölliker's organ decreased and their morphology changed from high columnar to short columnar in a basal to apical manner (Figure 2). However, until now, our understanding of Kölliker's organ is largely limited to the fact that it will disappear within a short period of time after birth.

Apoptosis is a process of programmed cell death that occurs in multicellular organisms. ${ }^{16}$ Caspases, a group of cysteine proteases, play active roles in the programmed cell death; ${ }^{17,18}$ for example, caspase- 3 is indispensable for the apoptotic cleavage. ${ }^{19}$ Caspase-3, transformed into cleaved caspase-3 which is an active form of caspase-3, upon the release of cytochrome c from mitochondria, triggers the apoptotic process through the cleavage of other proteins within a cell. ${ }^{19}$ A previous study ${ }^{8}$ has shown that in the capspase-3 knockout mice, Kölliker's organ persisted throughout all of the turns of the cochlea in 2-week-old mice, followed by hair cells degeneration and severe hearing loss at 5 weeks, suggesting the importance of caspase-3 in the maturation of cochlear structure in the early postnatal period. The activation of the caspase protein is regulated by a variety of factors including bcl-2 protein families. The anti-apoptosis protein bcl-2, a member of the bcl-2 families, stabilizes mitochondria membranes and thus inhibits apoptosis. ${ }^{20}$

In the present study, the triggering pathways converged on a common mechanism for the disappearance of Kölliker's organ, which was initiated by the caspase family of
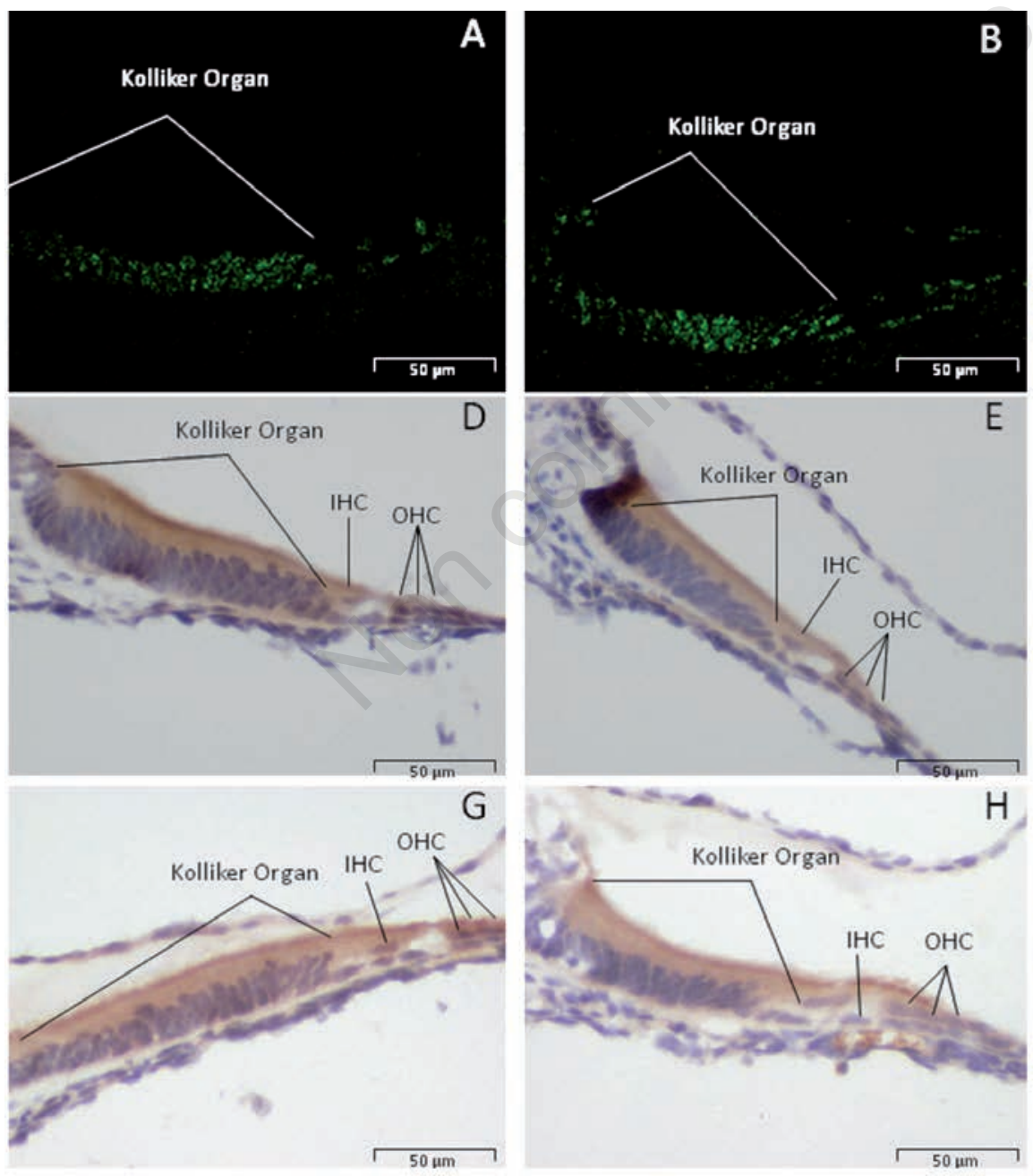
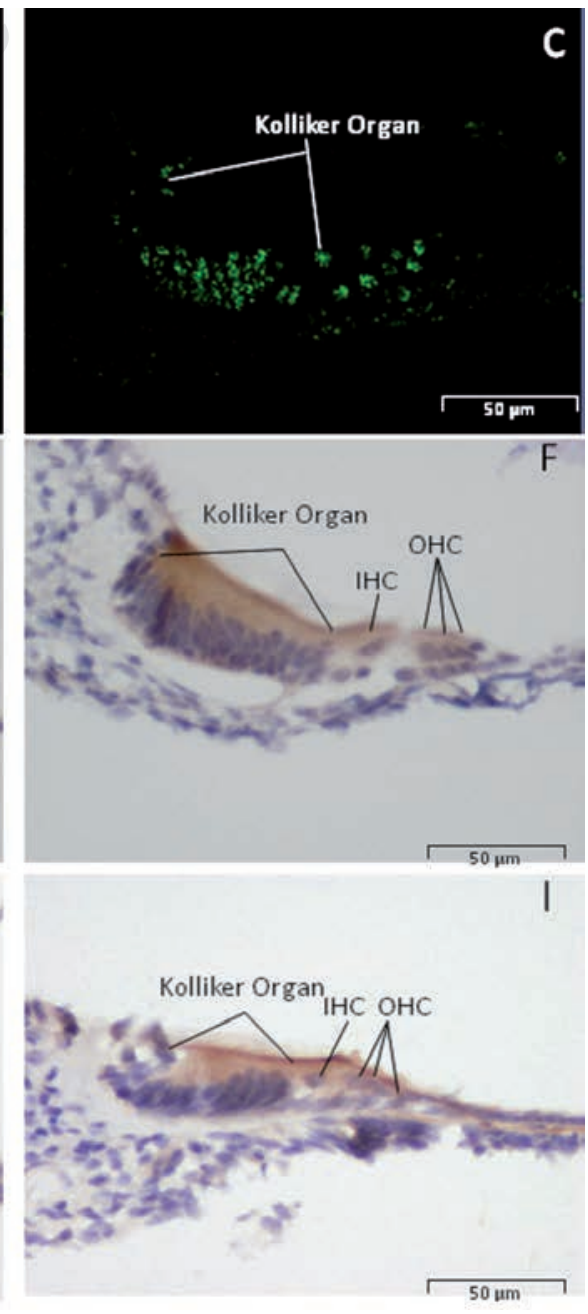

Figure 3. Immunohistochemistry and TUNEL stained sections from the apical, middle, and basal turns of the cochlea at P5 were displayed. A-C) TUNEL positive cells in Kölliker's organ stained green were observed in the apical, middle and basal turns of the cochlea. D-F) The bcl-2 protein expressed in the cytoplasm of SCs in Kölliker's organ from the apex turn to the basal turn at P5. G-I) The caspase-3 protein expressed in the cytoplasm of SCs in Kölliker's organ from the apex turn to the basal turn at P5. 
proteases. The caspse- 3 expressed at P1, suggesting that there might be apoptosis already in the embryonic stage, peaked at P3, and weakened at P12 in our present study, and the cleaved caspase-3 which, consistently showed a high expression in Kölliker's organ of the P1 and P3 rats, further indicating apoptosis in Kölliker's organ. Furthermore, the expression trend of bcl-2 was consistent with the above trends. Given these findings, it seems that apoptosis and anti-apoptosis present simultaneously in Kölliker's organ in vivo. Further research is needed to prove this. We speculated that not only SCs apoptosis but also proliferation happens in vivo. When the imbalance between apoptosis and antiapoptosis occurs, SCs present as apoptosis or proliferation. If the number of cell proliferation is far less than that of apoptotic cells, apoptosis appears on the macroscopic structure. Caspase-8, a key mediator in the extrinsic apoptotic pathway, is activated upon the binding of the death receptors on the cell membrane. ${ }^{21}$ Active caspase- 8 then either cleaves the downstream caspase-3 into cleaved caspase- 3 or triggers the cytochrome c release, thereby initiating the apoptotic cascades. As an initiator in the intrinsic pathway, caspase- 9 is cleaved into the active form and goes to activate caspase-3 upon the release of cytochrome c from mitochondria. ${ }^{22}$ In the present study, caspase-8, an upstream mediator of extracellular apoptotic initiator in the cell death pathways, had the strongest expression at P3. Moreover, caspase-9, an upstream mediator of intracellular apoptotic initiator, is highly expressed in P1-P10 rats without a peak expression. Therefore, we suggest that there are extrinsic and intrinsic apoptotic pathways during the apoptosis of SCs with the development of Kölliker's organ of the rat cochlea in vivo. Our results revealed the consistency of expression of the factors above in cochlear Kölliker's organs for the first time, and it is a preliminary understanding of the expression trend of these proteins in the development of the cochlea. However, further studies are needed to clarify the underlying mechanism.

In conclusion, Kölliker's organ in the cochlea degenerates after birth in a timedepending pattern along the cochlea apexto-base gradient, in which caspases are involved in the process of apoptotic cell death during postnatal development, suggesting involvement of endogenous factors. And there are studies learning about other endogenous factors such as Ngb immunoreactivity, which may participate in defense mechanisms in inner ear pathologies where oxidative stress is involved. ${ }^{23}$ Meanwhile,
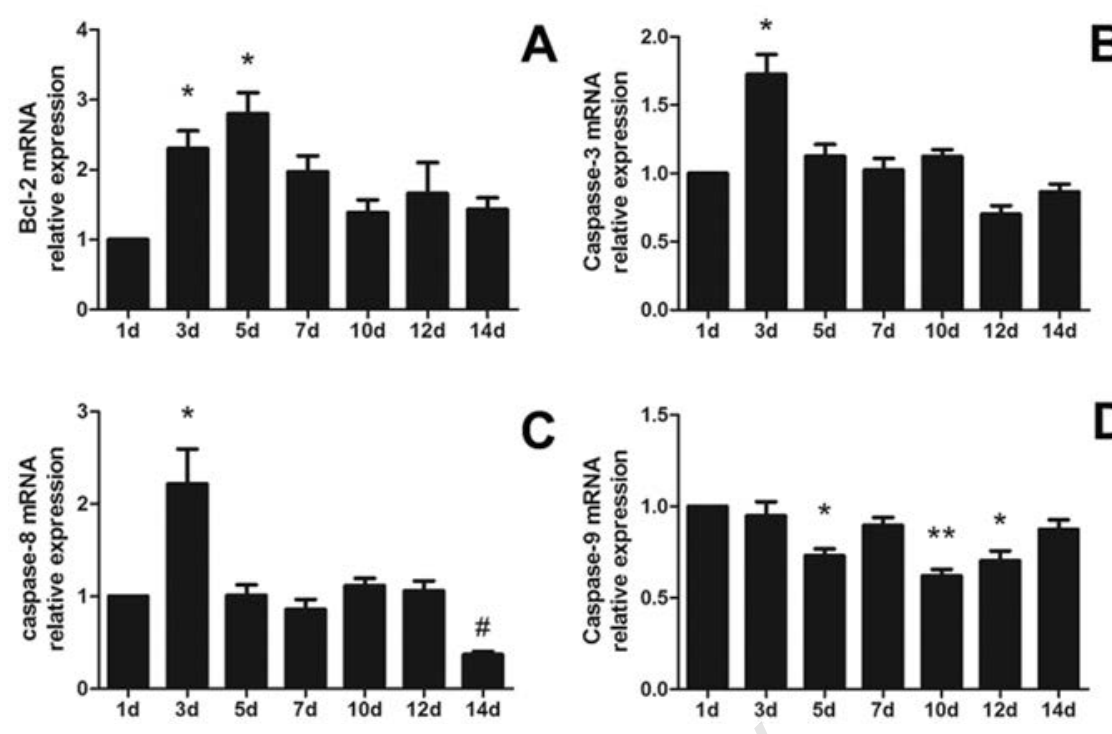

Figure 4. Realtime PCR analysis of bcl-2, caspase-3, caspase-8 and caspase-9 in Kölliker's organ in vivo. A-D) Realtime PCR analysis of relative mRNA expression levels of the bcl2 , caspase- 3 , caspase- 8 and caspase- 9 were used to estimate the apoptosis related-factors of the cochlear basilar membrane. Bar graph showed mean \pm SEM from each group with six repeats. ${ }^{*} \mathrm{P}<0.05$ compared with $\mathrm{P} 1,{ }^{* *} \mathbf{P}<\mathbf{0 . 0 5}$ compared with $\mathrm{P} 1, \# \mathbf{P}<\mathbf{0 . 0 5}$ compared with $P 1$, independent samples t-test.

\begin{tabular}{|c|c|c|c|c|c|c|}
\hline Bcl-2 & 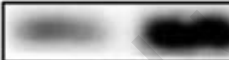 & 6. & 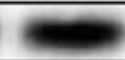 & 0 & -8 & $=$ \\
\hline & 2.26 & 2.18 & 2.15 & 1.14 & 1.17 & 0.85 \\
\hline pase-3 & 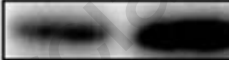 & 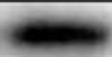 & 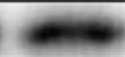 & -0 & 10 & 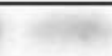 \\
\hline & 1.00 & 1.68 & 1.11 & 0.96 & 0.57 & 0.39 \\
\hline & $\mathrm{cos}$ & 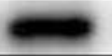 & 0 & 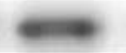 & 6 & $m$ \\
\hline & 1.00 & 1.96 & 1.47 & 0.98 & 0.78 & 0.66 \\
\hline & 1.00 & 1.23 & 1.34 & 0.95 & 0.55 & 0.49 \\
\hline & $3 d$ & $5 d$ & $7 d$ & $10 d$ & $12 d$ & $14 d$ \\
\hline
\end{tabular}

Figure 5. Western blot analysis of bcl-2, caspase-3, caspase- 8 and caspase- 9 in Kölliker's organ in vivo. Western blot analysis showed cytoplasmic proteins from the cochlear basilar membrane in P1, P3, P5, P7, P10, P12 and P14. Expression of proteins of Kölliker's organ at different time points during 1-14 days in vivo; a representative image of Western blots, which were performed using primary antibodies against bcl-2, caspase-3, caspase- 8 and caspase- 9 from each group. $\beta$-actin was used as an internal loading control.

\section{Cleaved Caspase-3}
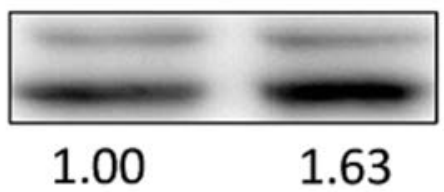

$\beta$-actin

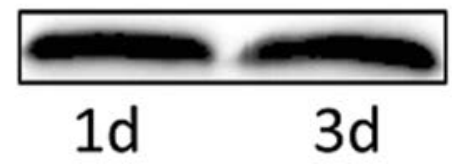

Figure 6. Western blot analysis of cleaved caspase-3 in P1 and P3 Kölliker's organ in vivo. The protein expression of cleaved caspase- 3 consistently showed a higher expression in Kölliker's organ of the $P 3$ rats than that of P1 rats. 
Lu et al. ${ }^{24}$ have found that the exogenous Wnt agonist BIO overcame the downregulation of SCs proliferation in Bmil-/mice, providing a possibility of exogeous factors involved. There is more work waiting for us.

\section{References}

1. Dayaratne MW, Vlajkovic SM, Lipski J, Thorne PR. Kolliker's organ and the development of spontaneous activity in the auditory system: implications for hearing dysfunction. Biomed Res Int 2014;2014:367939.

2. Anniko M. Postnatal maturation of cochlear sensory hairs in the mouse. Anat Embryol (Berl) 1983;166):355-68.

3. Tritsch NX, Yi E, Gale JE, Glowatzki E, Bergles DE. The origin of spontaneous activity in the developing auditory system. Nature 2007;450:50-5.

4. Sendin G, Bourien J, Rassendren F, Puel JL, Nouvian R. Spatiotemporal pattern of action potential firing in developing inner hair cells of the mouse cochlea. Proc Natl Acad Sci USA 2014;111:1999-2004.

5. He S, Yang J. Maturation of neurotransmission in the developing rat cochlea: immunohistochemical evidence from differential expression of synaptophysin and synaptobrevin 2. Eur J Histochem 2011;55:e2.

6. Kennedy HJ. New developments in understanding the mechanisms and function of spontaneous electrical activity in the developing mammalian audi- tory system. J Assoc Res Otolaryngol 2012;13:437-45.

7. Knipper M, Gestwa L, Ten Cate WJ, Lautermann J, Brugger H, Maier H, et al. Distinct thyroid hormone-dependent expression of $\operatorname{TrKB}$ and $\mathrm{p} 75 \mathrm{NGFR}$ in nonneuronal cells during the critical TH-dependent period of the cochlea. J Neurobiol 1999;38:338-56.

8. Kamiya K, Takahashi K, Kitamura K, Momoi T, Yoshikawa Y. Mitosis and apoptosis in postnatal auditory system of the $\mathrm{C} 3 \mathrm{H} / \mathrm{He}$ strain. Brain Res 2001;901:296-302.

9. Raff M. Cell suicide for beginners. Nature 1998;396:119-22.

10. Miller DK. The role of the Caspase family of cysteine proteases in apoptosis. Semin Immunol 1997;9:35-49.

11. Nicholson DW, Thornberry NA. Caspases: killer proteases. Trends Biochem Sci 1997;22:299-306.

12. Liu WJ, Yang J. Preferentially regulated expression of connexin 43 in the developing spiral ganglion neurons and afferent terminals in post-natal rat cochlea. Eur J Histochem 2015;59: 2464.

13. Liu WJ, Yang J. Developmental expression of inositol 1, 4, 5-trisphosphate receptor in the post-natal rat cochlea. Eur J Histochem 2015;59:2486.

14. Hinojosa R. A note on development of Corti's organ. Acta Otolaryngol 1977;84:238-51.

15. Tritsch NX, Zhang YX, Ellis-Davies G, Bergles DE. ATP-induced morphological changes in supporting cells of the developing cochlea. Purinergic Signal
2010;6:155-66.

16. Green DR. Means to an end: apoptosis and other cell death mechanisms. Cold Spring Harbor Laboratory Press, Cold Spring Harbor, New York; 2011.

17. Leon Y, Sanchez-Galiano S, Gorospe I. Programmed cell death in the development of the vertebrate inner ear. Apoptosis 2004;9(255-64.

18. Nicholson DW. Caspase structure, proteolytic substrates, and function during apoptotic cell death. Cell Death Differ 1999;6:1028-42.

19. Janicke RU, Ng P, Sprengart ML, Porter AG. Caspase-3 is required for alphafodrin cleavage but dispensable for cleavage of other death substrates in apoptosis. J Biol Chem 1998;273: 15540-5.

20. Vaux DL. A boom time for necrobiology. Curr Biol 1993;3:877-8.

21. Creagh EM. Caspase crosstalk: integration of apoptotic and innate immune signalling pathways. Trends Immunol 2014;35:631-40.

22. Adrain C, Martin SJ. The mitochondrial apoptosome: a killer unleashed by the cytochrome seas. Trends Biochem Sci 2001;26:390-7.

23. Vorasubin N, Hosokawa S, Hosokawa $\mathrm{K}$, Ishiyama G, Ishiyama A, Lopez IA. Neuroglobin immunoreactivity in the human cochlea. Brain Res 2016;1630: 56-63.

24. Lu X, Sun S, Qi J, Li W, Liu L, Zhang $\mathrm{Y}$, et al. Bmil regulates the proliferation of cochlear supporting cells via the canonical Wnt signaling pathway. Mol Neurobiol 2017;54:1326-39. 